\title{
Multidimensional SAR Data Analysis Based on Binary Partition Trees and the Covariance Matrix Geometry
}

\author{
Alberto Alonso-González \\ Carlos López-Martínez \\ Philippe Salembier \\ Signal Theory and Comms. Dept. (TSC) \\ Universitat Politècnica de Catalunya (UPC) Email: silvia.valero@cesbio.cnes.fr \\ Barcelona, Spain \\ Email: \{carlos.lopez,alberto.alonso,philippe\} \\ @ tsc.upc.edu \\ Silvia Valero \\ CESBIO - CNES \\ CNRS (UMR 5126), IRD \\ Universite de Toulouse, France

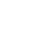

Jocelyn Chanussot

Grenoble Institute of Technology

Grenoble Images sPeech

Signals and Automatics Lab (GIPSA-Lab)

Grenoble, France

Email: jocelyn.chanussot

@ gipsa-lab.grenoble-inp.fr

\begin{abstract}
In this paper, we propose the use of the Binary Partition Tree (BPT) as a region-based and multi-scale image representation to process multidimensional SAR data, with special emphasis on polarimetric SAR data. We also show that this approach could be extended to other types of remote sensing imaging technologies, such as hyperspatial imagery. The Binary Partition Tree contains a lot of information about the image structure at different detail levels. At the same time, this structure represents a convenient vehicle to exploit both the statistical properties, as well as the geometric properties of the multidimensional SAR data given by the covariance matrix. The BPT construction process and its exploitation for PolSAR and temporal data information estimation is analyzed in this work. In particular, this work focuses on the speckle noise filtering problem and the temporal characterization of the image dynamics. Results with real data are presented to illustrate the capabilities of the BPT processing approach, specially to maintain the spatial resolution and the small details of the image.
\end{abstract} Tree.

Keywords-SAR, PolSAR, speckle filtering, Binary Partition

\section{INTRODUCTION}

Polarimetric SAR (PolSAR) and multitemporal SAR data have demonstrated their significance for the analysis and the characterization of the Earth surface, as well as for the quantitative retrieval of biophysical and geophysical parameters. A set of complex radar scattered echoes are coherently processed in order to achieve high spatial resolution. As a consequence of this coherent processing and the fact that each resolution cell is a combination of a certain number of elementary echoes, the received signal is affected by the speckle term. Despite speckle is determined by the scattering process itself, its complexity makes it necessary to consider it from a stochastic point of view and then, to assume the speckle term as a noise component. The speckle is a handicap in SAR imagery processing and consequently some speckle filtering process is needed. Under the assumption that multidimensional SAR data are distributed according to the zero-mean, complex Gaussian distribution, the objective of this filtering process is to obtain, i.e., to estimate the covariance matrix characterizing the multidimensional data.

Multidimensional SAR data are non stationary as they reflect the complexity of the environment. Assuming that all the stochastic processes involved in the filtering process are ergodic, multidimensional SAR data filters must adapt to this non stationarity [1], as well as to the geometry of the covariance matrix. Most recent state-of-the-art filtering techniques [2], [3] are based on this approach, tending to define a different homogeneity neighbourhood for each image pixel. In this paper, we propose to tackle this issue by relying on an image region-based multi-scale representation by means of a Binary Partition Tree (BPT) [4] and to perform the image processing, in terms of filtering or segmentation tasks, directly by pruning the BPT. Since the BPT operates in the space defined by the covariance matrix, this processing approach may take into account the matrix geometry by defining a proper metric. In this work, we focus into two central applications: PolSAR data speckle filtering and the temporal characterization of temporal series of PolSAR data. Finally, we also demonstrate that this approach can be also applied in the case of hyperspectral data.

\section{STATISTICAL CHARACTERIZATION OF MULTIDIMENSIONAL SAR DATA}

A multidimensional SAR system records a set of $m$ SAR images, represented by the target vector

$$
\mathbf{k}=\left[S_{1}, S_{2}, \ldots, S_{m}\right]^{T}
$$

where $S_{k}$, for $k=1,2, \ldots, m$, represents each one of the complex SAR images and ${ }^{T}$ denotes the vector transposition. The gain which can be achieved by a multidimensional SAR system, respect to a one-dimensional one, results from the fact that the same scattering mechanism is sampled $m$ times under some type of diversity. One of the best examples of multidimensional SAR data corresponds to PolSAR data, where the diversity is achieved by changing the polarization of the transmitted field and the receiving antenna. Another example of multidimensional SAR data is the acquisition of temporal series of data over the same ares of observation. As it may be 
observed, both sources of diversity may be combined together. Most of the PolSAR systems alternate between horizontal and vertical polarization states. Under the hypothesis that the different SAR images are able to be described by means of zero-mean, complex, Gaussian distributions, the target vector $\mathbf{k}$ has the following distribution

$$
p_{\mathbf{k}}(\mathbf{k})=\frac{1}{\pi^{m}|\mathbf{C}|} \exp \left(-\mathbf{k}^{H} \mathbf{C}^{-1} \mathbf{k}\right)
$$

where ${ }^{H}$ refers to the transpose complex conjugation and $\mathbf{C}$ is the positive definite, Hermitian covariance matrix

$$
\begin{aligned}
\mathbf{C} & =E\left\{\mathbf{k k}^{H}\right\} \\
& =\left[\begin{array}{cccc}
E\left\{S_{1} S_{1}^{*}\right\} & E\left\{S_{1} S_{2}^{*}\right\} & \cdots & E\left\{S_{1} S_{m}^{*}\right\} \\
E\left\{S_{2} S_{1}^{*}\right\} & E\left\{S_{2} S_{2}^{*}\right\} & \cdots & E\left\{S_{2} S_{m}^{*}\right\} \\
\vdots & \vdots & \ddots & \vdots \\
E\left\{S_{m} S_{1}^{*}\right\} & E\left\{S_{m} S_{2}^{*}\right\} & \cdots & E\left\{S_{m} S_{m}^{*}\right\}
\end{array}\right] .
\end{aligned}
$$

Since $E\{\mathbf{k}\}=\mathbf{0}$, the information of interest about the area illuminated by the radar is contained in the matrix $\mathbf{C}$. Hence, this matrix needs to be estimated from the original data (1). As observed in (3), the information is not only contained in the SAR images intensities, but also on the correlation structure given by the off-diagonal complex elements of (3).

The process to estimate $\mathbf{C}$ is referred to as the multidimensional speckle reduction process. The estimation is performed from the so-called one-look, sample covariance matrix $\mathbf{Z}$. The most standard approach to estimate $\mathbf{C}$ is the multilook technique

$$
\mathbf{Z}_{n}=\frac{1}{n} \sum_{i=1}^{n} \mathbf{Z}_{i}=\frac{1}{n} \sum_{i=1}^{n} \mathbf{k}_{i} \mathbf{k}_{i}^{H}
$$

where $\mathbf{Z}_{i}$ and $\mathbf{k}_{i}$ correspond, respectively, to the single-look sample covariance matrix and the target vector of the $i$-th pixel, out of $n$, employed to estimate $\mathbf{C}$ from the total number of samples $n$. The main drawback of (4) is that $\mathbf{C}$ is estimated at the expense of spatial resolution and spatial details. In order to overcome this problem, other approaches have been presented in the literature to estimate $\mathbf{C}$. Considering (4), and under the hypothesis that $n>m$, the matrix $\mathbf{Z}_{n}$ is described by means of a Wishart distribution $\mathcal{W}(\mathbf{C}, n)$ [5][6]

$$
p_{\mathbf{Z}}(\mathbf{Z})=\frac{n^{3 n}|\mathbf{Z}|^{n-3}}{|\mathbf{C}|^{n} \pi^{3} \prod_{i=1}^{3} \Gamma(n-i+1)} \operatorname{etr}\left(-n \mathbf{C}^{-1} \mathbf{Z}\right)
$$

where $\operatorname{etr}(\mathbf{X})$ is the exponential of the matrix trace and $n \geq 3$.

\section{BINARY PARTITION TREE}

The Binary Partition Tree (BPT) was introduced in [4] as a region-based and multi-scale image representation. It contains information of the image structure at different detail levels within a tree. In this hierarchical structure, each node represents a connected region of the image. The tree leaves represent single pixels and all the other nodes represent the region composed by merging its two child nodes. Finally, the root node represents the whole image. Thus, the tree edges describe the inclusion relationship between nodes. Between the leafs and the root there are a wide number of nodes representing image regions with different detail level. This multi-scale representation contains a lot of useful information about the image structure that can be exploited for different applications.

In order to be able to exploit the information provided by the Wishart distribution in (5), one must account for the limitation that $n \geq 3$. When the covariance matrix is constructed in a pixel basis, as the hermitian product $\mathbf{k}_{i} \mathbf{k}_{i}^{H}$, the resulting matrix is of rank one which prevents the use of the Wishart distributuon. In order to solve it, the covariance matrices at pixel level need to be regularized to assure rank three matrices. This regularization may be obtained by means of a 3 times 3 multilook filter. In order to reduce the loss of spatial resolution, a bilateral filter may be also considered [7].

To construct the BPT representation from an image, an iterative algorithm is employed in a bottom-up approach [4]. In the initial state, every pixel of the image becomes a onepixel region. At every step, the two most similar regions are merged and this process is repeated until the root of the tree, containing the whole image, is generated. In order to apply this algorithm, two important concepts need to be determined [8]:

1) A region model: Traditionally, under the complex Gaussian model, the estimated covariance matrix $\mathbf{Z}$ is employed to measure the region polarimetric information, see (4). Additionally, since during the BPT construction process regions of different sizes coexist, the region size information should be taken into account and will be included in the region model.

2) A similarity measure on the region model space to compare two neighboring regions $d(X, Y)$. Two types of measures will be analyzed in this work. On the one hand measures based on the statistical distribution and on the other hand measures based on the covariance matrix subspace geometry.

The revised Wishart measure [9] $d_{w}$ is based on a statistical test assuming Wishart distributions and that one region statistics are known. However, since this measure is not symmetric, a modified symmetric version will be applied

$$
\begin{gathered}
d_{s w}(X, Y)=\left(\operatorname{tr}\left(\mathbf{Z}_{X}^{-1} \mathbf{Z}_{Y}\right)+\operatorname{tr}\left(\mathbf{Z}_{Y}^{-1} \mathbf{Z}_{X}\right)\right) . \\
\cdot\left(n_{x}+n_{y}\right)
\end{gathered}
$$

where $\operatorname{tr}($.$) represents the matrix trace, \mathbf{Z}_{X}$ and $\mathbf{Z}_{Y}$ represent the estimated covariance matrices for regions $X$ and $Y$, respectively, and $n_{x}$ and $n_{y}$ represent their number of pixels.

For comparison purposes a new version of the symmetric revised Wishart dissimilarity will be used, only taking into account the diagonal elements of the $\mathbf{Z}$ matrix and assuming all off-diagonal values equal to zero

$$
d_{d w}(X, Y)=\left(\sum_{i=1}^{3}\left(\frac{Z_{X}^{2}+Z_{Y i i}^{2}}{Z_{X i i} Z_{Y i i}}\right)\right) \cdot\left(n_{x}+n_{y}\right)
$$

where $Z_{X i j}$ and $Z_{Y i j}$ represent the (i,j)-th element of the estimated covariance matrices $\mathbf{Z}_{X}$ and $\mathbf{Z}_{Y}$, respectively. It is interesting to note that since this distance only considers the information provided only by the diagonal elements of the covariance matrix, that is, by construction it assumes diagonal matrices 
of rank three, an initial regularization process is not necessary.

Another family of dissimilarities that is analyzed are based on the positive definite matrix cone geometry [10]

$$
\begin{aligned}
d_{s g}(X, Y)=\| \log ( & \left.\mathbf{Z}_{X}^{-1 / 2} \mathbf{Z}_{Y} \mathbf{Z}_{X}^{-1 / 2}\right) \|_{F}+ \\
& +\ln \left(\frac{2 n_{x} n_{y}}{n_{x}+n_{y}}\right)
\end{aligned}
$$

where $\|.\|_{F}$ represents the Frobenius matrix norm, $\log ($.$) represents the matrix logarithm and \ln ($.$) rep-$ resents the natural logarithm.

As for the Wishart dissimilarities, a new version is defined employing only the information contained in the diagonal elements of the covariance matrix

$$
d_{d g}(X, Y)=\sqrt{\sum_{i=1}^{3} \ln ^{2}\left(\frac{Z_{X i i}}{Z_{Y i i}}\right)}+\ln \left(\frac{2 n_{x} n_{y}}{n_{x}+n_{y}}\right)
$$

Again in this case, no initial regularization is needed as the the distance, by constructions, assumes ranks three matrices.

\section{BPT PRUNING}

The BPT is a hierarchical representation of the image structure at different details levels. Thus, it depends only on the image and consequently it is application independent. One possible approach to develop BPT-based applications is to select a set of meaningful regions within the tree. As mentioned in [4], an image segmentation could be obtained by tree pruning.

The BPT punning process policy depends of the final application. For the PolSAR speckle noise filtering application, the main objective is to obtain the biggest possible homogeneous regions of the image. The BPT and its multi-scale nature can be exploited for this application. Then, an homogeneity-based tree pruning can be performed. A region homogeneity measure $\phi$ has to be defined to be able to define a pruning process. In [11], the following criterion based on the Frobenius matrix norm is proposed

$$
\phi_{R}(X)=\frac{1}{n_{x}} \sum_{i=1}^{n_{x}} \frac{\left\|\mathbf{X}^{i}-\mathbf{Z}_{X}\right\|_{F}^{2}}{\left\|\mathbf{Z}_{X}\right\|_{F}^{2}}
$$

where $\mathbf{X}^{i}$ is the estimated covariance matrix for the $\mathrm{i}$-th pixel within region $X$.

Note that this measure depends on all the pixel values within the $X$ region and not only on its model, as the dissimilarity measure. Additionally, $\phi_{R}$ is independent of the region size, since it is an average over all the region pixels. This is an important property of the homogeneity measure in order to define the region homogeneity independently of its size. The measure (10) can be seen as the mean information loss when modeling all the pixels within a region with its estimated covariance matrix. Finally, to determine if a region is homogeneous or not, a maximum value $\delta_{p}$ for the homogeneity measure has to be defined, called pruning threshold. Then, the nodes of the tree are scanned from the top to the leaves and the punning is done at the first node, that represents the region $X_{i}$, having $\phi_{R}\left(X_{i}\right)<\delta_{p}$. Consequently, this punning selects the biggest homogeneous regions from the tree. In this paper $\delta_{p}$ will be expressed in $\mathrm{dB}$ scale, corresponding to $10 \cdot \log _{10}\left(\phi_{R}\right)$.

\section{Polsar Speckle Filtering Results}

The described homogeneity based BPT pruning for PolSAR speckle noise filtering has been tested with a real PolSAR dataset. Fig. 1 shows the image corresponding to PolSAR data that was acquired in a measurement campaign conducted by the DLR in 1999 with its experimental SAR system, ESAR, over the Oberpfaffenhofen test-site, southern Germany. Data were collected at L-band, with a spatial resolution of $1.5 m \times 1.5 m$ in fully linear polarimetric mode.

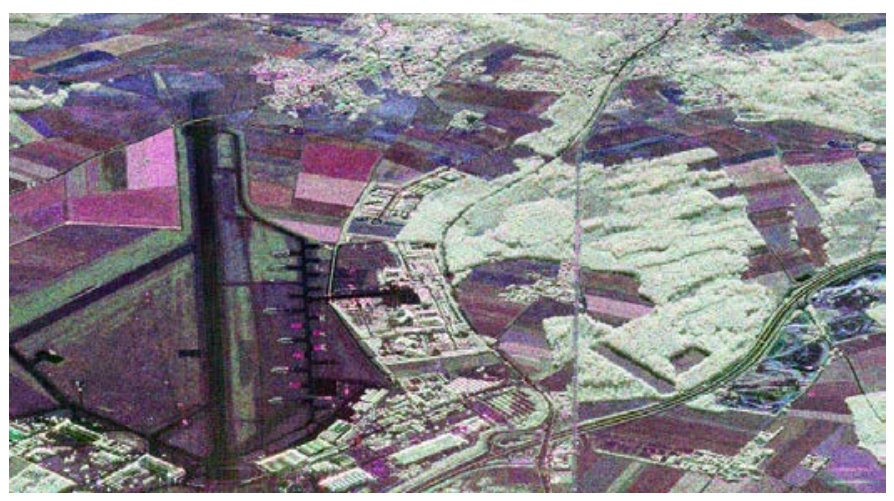

Fig. 1: Pauli RGB (R: Double bounce, G: Volume, B: Single bounce) coding of the original E-SAR image.

On Fig. 5, different pruning results are shown over the same BPT constructed with the $d_{s w}$ dissimilarity (6). Increasing the pruning threshold $\delta_{p}$ results into bigger regions of the pruned tree, as less homogeneous regions are accepted.

It is worth to notice that in the same image there are regions with very different sizes. Large homogeneous areas, as the agricultural fields in the left part of the image, whereas point scatterers or details from the urban area in the center of the image are preserved as very small regions.

A $512 \times 512$ pixels area of the original data is selected to see a more detailed view of the results. The $7 \times 7$ multilook is shown in Fig. $3 b$ for comparison purposes. In Figs. 3c$3 \mathrm{f}$ different results are shown corresponding to the same tree pruning process over different BPTs constructed with the different similarity measures proposed in the previous section. If compared with the $7 \times 7$ multilook, the BPT based filtering preserves much better original image spatial resolution and spatial details. Comparing the results obtained with the different similarity measures presented in the previous section one may observe that there are very subtle differences between the results. This similarity of results allows to demonstrate that the BPT representation tool is very robust respect to the similarity measure employed for the BPT construction process. In addition, is is important to notice that the colours of the regions, which depends on the physical scattering process, does not change respect to the original image. This maintenance of the physical information indicates that a part of the robustness, the BPT representation approach does not degrades the physical information content of the data. 


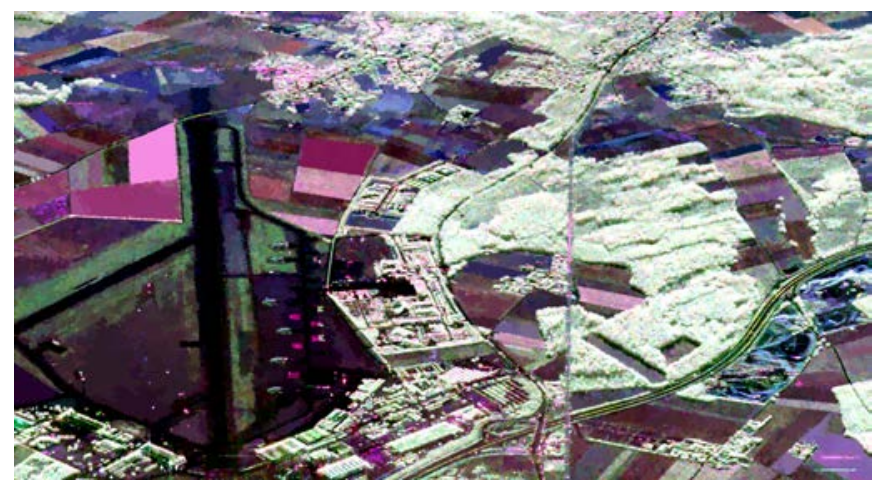

(a) $\delta_{p}=-2 d B$

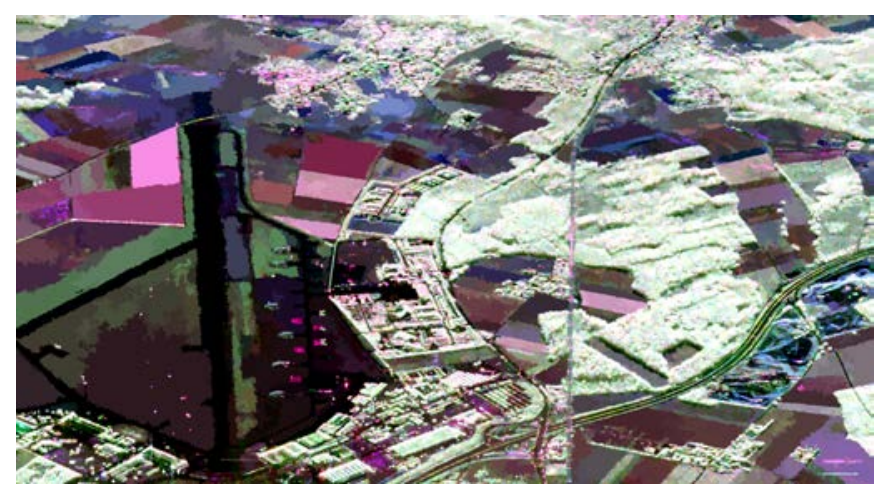

(b) $\delta_{p}=-1 d B$

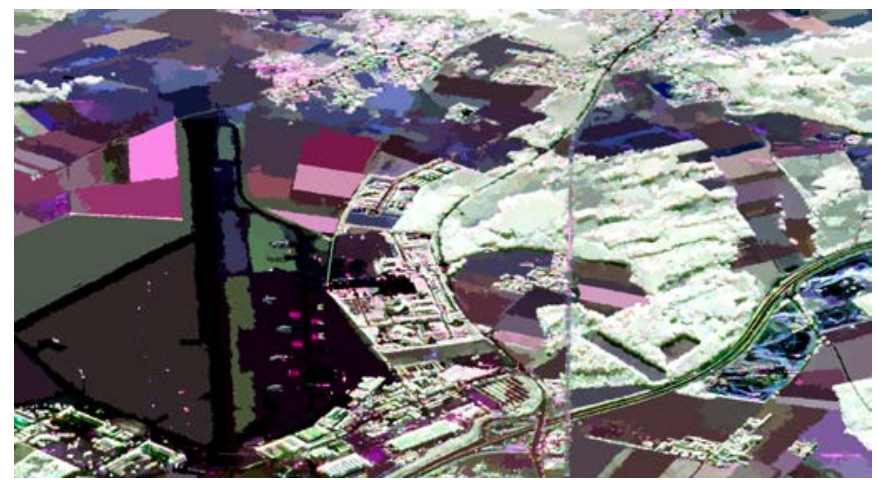

(c) $\delta_{p}=0 d B$

Fig. 2: Pauli RGB (R: Double bounce, G: Volume, B: Single bounce) processed images.

\section{TEMPORAL INFORMATION ESTIMATION}

In the previous sections, the BPT has been employed to represent PolSAR data for a single temporal acquisition. Nevertheless, a SAR system can also image the same area of observation at different times, being able to provide a temporal series of data. Every single acquisition may contain $m$ complex SAR images, see (1), so each of the acquisitions can also present polarimetric diversity. Consequently, if we consider PolSAR data, the incorporation of temporal diversity adds an additional dimension to every pixel consisting on time.

In the previous scenario of increased dimensionality, the BPT can be also considered as a tool to represent the data in a hierarchical structure. Nevertheless, the regions will be no

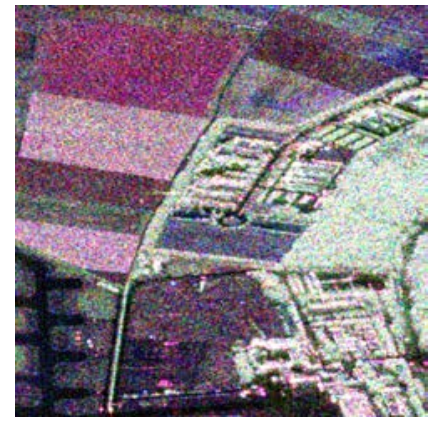

(a) Original

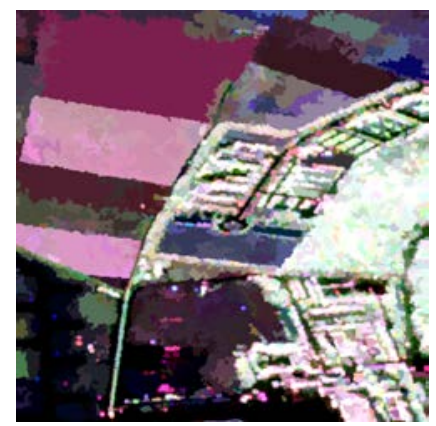

(c) $d_{d w}, \delta_{p}=-1 d B$

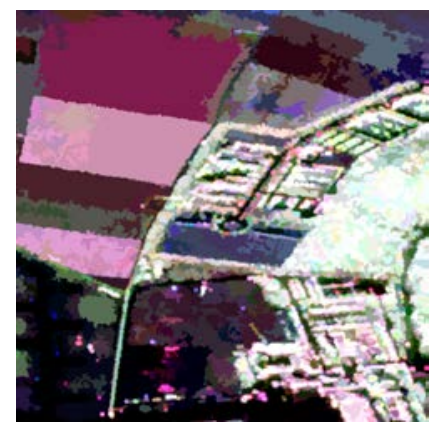

(e) $d_{d g}, \delta_{p}=-1 d B$

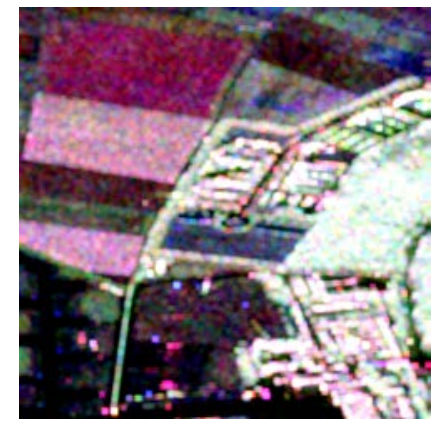

(b) $7 \times 7$ multilook

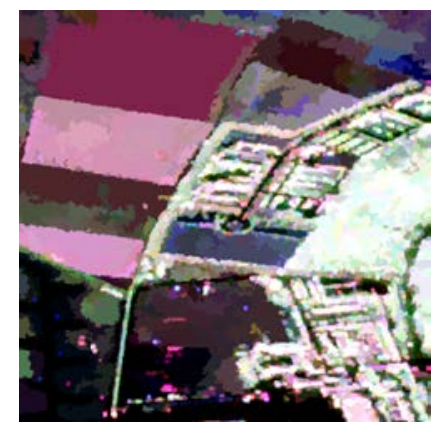

(d) $d_{s w}, \delta_{p}=-1 d B$

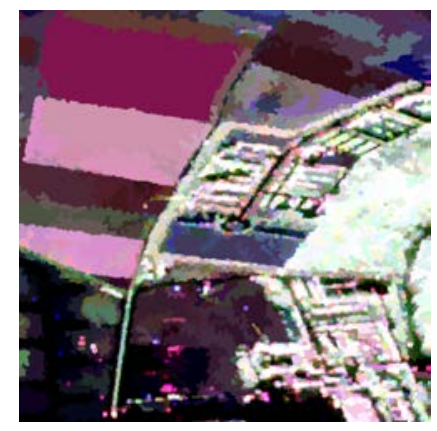

(f) $d_{s g}, \delta_{p}=-1 d B$
Fig. 3: Detail Pauli RGB (R: Double bounce, G: Volume, B: Single bounce) original and BPT processed images with $\delta_{p}=-1 d B$ over different trees constructed employing various similarity criteria.

longer two dimensional spatial regions, but three dimensional space-time regions. Consequently, it is necessary to adapt the BPT structure to include the temporal dimension information by adapting: the region model, the similarity measure and the homogeneity criteria. Regarding the region model, there exit two options to include temporal information.

A first option to include temporal information is to maintain the regions models as the $3 \times 3$ estimated covariance matrix $\mathbf{Z}$ [12].Thus, the temporal dimension represents an additional data dimension. Hence, the BPT contains connected regions of the data in the space-time domain having similar estimated covariance matrix $\mathbf{Z}$. A second approach that may be considered is to consider the time information within the region model. This different point of view allows to assume the variation of the polarimetric information, i.e., the temporal 
signature of the target, as an intrinsic characteristic or property of the target itself. In order to include the time information in the characterization of the target results in the augmented covariance matrix $\mathbf{Z}_{N}$

$$
\mathbf{Z}_{N}=\left(\begin{array}{cccc}
\mathbf{Z}_{11} & \boldsymbol{\Omega}_{12} & \cdots & \boldsymbol{\Omega}_{1 N} \\
\boldsymbol{\Omega}_{12}^{H} & \mathbf{Z}_{22} & \cdots & \boldsymbol{\Omega}_{2 N} \\
\vdots & \vdots & \ddots & \vdots \\
\boldsymbol{\Omega}_{1 N}^{H} & \boldsymbol{\Omega}_{2 N}^{H} & \cdots & \mathbf{Z}_{N N}
\end{array}\right)
$$

where $N$ represents the number of temporal acquisitions, $\mathbf{Z}_{i i}$ is a 3 by 3 covariance matrix representing the polarimetric information of the $i$-th acquisition and $\boldsymbol{\Omega}_{i j}$ is a 3 by 3 complex matrix representing the interferometric information among the acquisitions $i$ and $j$. This approach generates a tree containing spatial connected regions of the data set and will produce region contours constant along time. In conclusion, by assuming this extended model a node will represent a group of pixels presenting similar temporal evolution.

In order to adapt the different distances to consider the region model introduced in (11), we propose to consider only the information provided by the polarimetric covariance matrices $\mathbf{Z}_{i i}$. We do not consider the information provided by the off-diagonal matrices $\mathbf{Z}_{i j}$ as this matrices contain interferometric information that may produce irregular regions not adapted tho the signal spatial morphology. In the case of the geodesic distance proposed in (8), the extension is assumed as

$$
d_{g}(X, Y)=\sum_{i=1}^{N} d_{s g}\left(\mathbf{Z}_{X_{i i}}, \mathbf{Z}_{Y_{i i}}\right) .
$$

Finally, the homogeneity criteria presented in (10) can be also consider temporal information by assuming the previous extension of the similarity criteria, i.e.,

$$
\phi_{M}(X)=\frac{1}{n_{x}} \sum_{i=1}^{n_{x}} \frac{\sum_{j=1}^{N}\left\|\mathbf{Z}_{j j}^{i}-\mathbf{Z}_{X_{j j}}\right\|_{F}^{2}}{\sum_{j=1}^{N}\left\|\mathbf{Z}_{X_{j j}}\right\|_{F}^{2}}<\delta_{p} .
$$

To demonstrate the validity of this extension, a RADARSAT-2 Fine Quad-Pol (FQ13 ascending pass) dataset over Flevoland, the Netherlands is considered. The scene is composed by an area of agricultural fields with some sea surface and urban areas. The dataset contains 8 images from April 4th, 2009 to September 29th, 2009 with an acquisition every 24 days, see Fig. 4.

The extension of the BPT into the temporal dimension has been employed to process the previous dataset, see Fig. 4, as it can be observed in Fig.

To simplify the representation only the results over the second acquisitions are shown. As one may observe, the extension of the BPT still maintains the properties indicated in the previous section as the spatial resolution and the spatial details, as well as the physical information, represented by the colour, are preserved.

\section{PROCESSING HYPERSPECTRAL IMAGERY USING BPT}

As presented, BPT representation possesses some interesting features to deal with PolSAR times series images. Nevertheless, it must be remarked that BPT is not a specific

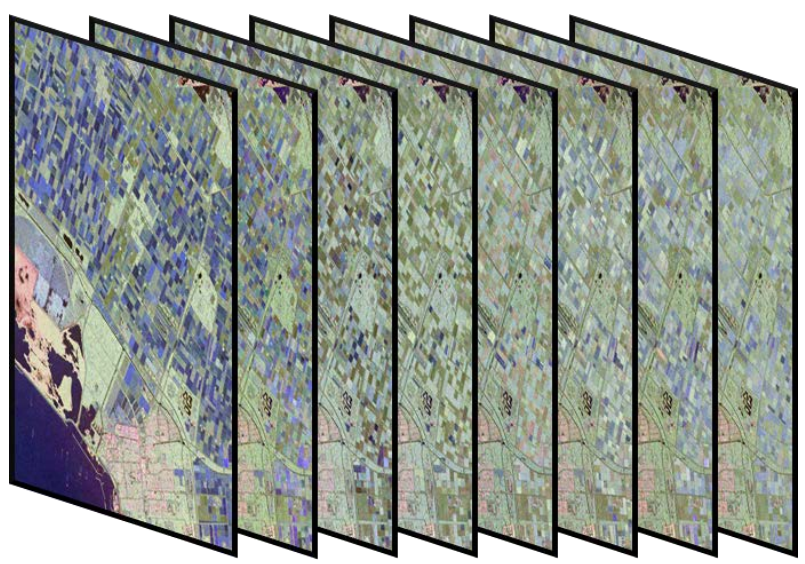

Fig. 4: Full dataset with 8 temporal acquisitions.

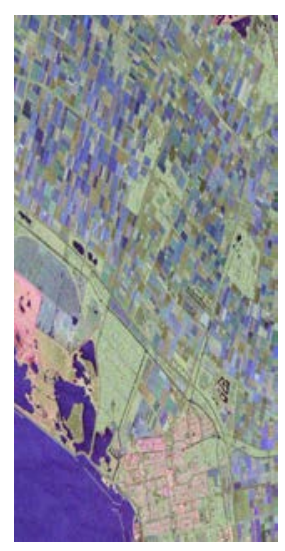

(a) Original

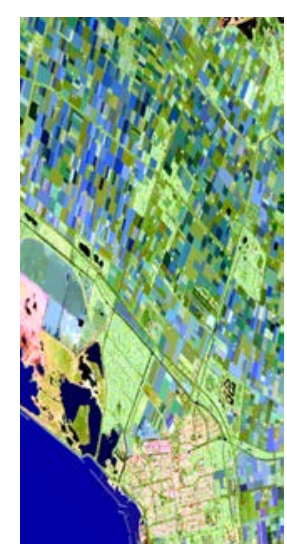

(b) Extended BPT, $\phi_{M}$
Fig. 5: Pauli representation for the second acquisition processed with the BPT with extended model. Results are shown for $\delta_{p}=-3 d B$.

tool for this type of data. Note that BPT can be built and employed to process another remote sensing data coming from a different signal nature, as for instance hyperspectral imagery (HSI).

A hyperspectral image is typically a compilation of a few hundred spectral images captured at different wavelengths. For this data, BPT representation can also be computed in order to define processing strategies dealing with many applications such as filtering, segmentation [13], classification [14], and object detection [15]. As indicated, the BPT construction is mainly based on the region merging algorithm definition. Concerning HSI data, the definition of a region model and a merging criterion has been previously studied in [14]. This work proposes as region model the set of normalized histograms of the pixels belonging to each region for each spectral band [16]. Concerning the similarity measure, the principal coordinates of each region are extracted from local dimensional reduction technique MDS and evaluated by using the classical Wilk's Statistical Test. Once BPT is constructed, this representation defines a search space which can be used for instance to construct a robust object identification scheme. In 
it, the spatial and spectral information are integrated in order to analyze hyperspectral images with a region-based perspective. In the context of object detection, this representation is a very powerful tool, since spatial and spectral descriptors can be computed for each region. Therefore, a likelihood distance can be evaluated in other to measure if a region correspond to an instantiation of the object of interest. The experimental results shown in Fig. 6 demonstrate the good performances of this BPT-based approach. In this example, the goal is to detect the buildings on HYDICE hyperspectral data containing 167 spectral bands and a spatial resolution of $3 \mathrm{~m}$.

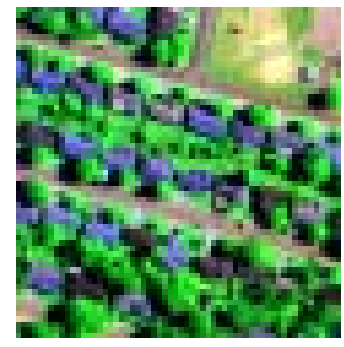

(a) HSI Color composition

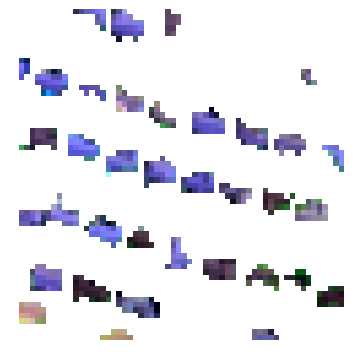

(b) Building detection result
Fig. 6: Building detection example using BPT-based detection.

\section{CONCLUSIONS}

As it has been demonstrated in this work, Binary Partition Trees (BPTs) are a powerful multiscale representation tool for multitemporal polarimetric SAR (PolSAR) data. This work has considered the application of BPTs for PolSAR data speckle noise filtering, as well as for the estimation of temporal information for multitemporal acquisitions. As shown, BPTs allow an easy inclusion of the concept of distance to differentiate different regions of the data. Among the different distances comparing different homogeneous areas represented by covariance matrices, the geodesic distance has shown the best performance. Nevertheless, the small differences obtained with other distances support the idea that BPTs are robust. Finally, BPTs have been also applied to hyperspectral imagery supporting their versatility.

\section{ACKNOWLEDGEMENTS}

This work has been funded by the MICINN TEC Project MUSEO (TEC2011-28201-C02-01). Data provided by the German Aerospace Center (DLR) and European Space Agency (ESA) in teh frame of the AGRISAR 2009 project.

\section{REFERENCES}

[1] S. Foucher and C. López-Martínez, "Analysis, evaluation, and comparison of polarimetric sar speckle filtering techniques," Image Processing, IEEE Transactions on, vol. 23, no. 4, pp. 1751-1764, April 2014.

[2] J.-S. Lee, M. Grunes, and G. de Grandi, "Polarimetric SAR speckle filtering and its implication for classification," Geoscience and Remote Sensing, IEEE Transactions on, vol. 37, no. 5, pp. 2363-2373, Sept. 1999.

[3] G. Vasile, E. Trouve, J.-S. Lee, and V. Buzuloiu, "Intensity-driven adaptive-neighborhood technique for polarimetric and interferometric SAR parameters estimation," Geoscience and Remote Sensing, IEEE Transactions on, vol. 44, no. 6, pp. 1609-1621, June 2006.
[4] P. Salembier and L. Garrido, "Binary partition tree as an efficient representation for image processing, segmentation, and information retrieval," Image Processing, IEEE Transactions on, vol. 9, no. 4, pp. 561-576, Apr 2000.

[5] N. R. Goodman, "Statistical analysis based on a certain multivariate complex gaussian distribution (an introduction)," Ann. Mathemat. Statist., vol. 34, pp. 152 - 177, 1963.

[6] C. Oliver and S. Quegan, Understanding Synthetic Aperture Radar Images. Boston, USA: Artech House, 1998.

[7] A. Alonso-Gonzlez, C. Lpez-Martnez, P. Salembier, and X. Deng, "Bilateral distance based filtering for polarimetric sar data," Remote Sensing, vol. 5, no. 11, pp. 5620-5641, 2013. [Online]. Available: http://www.mdpi.com/2072-4292/5/11/5620

[8] A. Alonso-Gonzalez, C. López-Martínez, and P. Salembier, "Filtering and segmentation of polarimetric SAR images with binary partition trees," in Geoscience and Remote Sensing Symposium (IGARSS), 2010 IEEE International, july 2010, pp. 4043 -4046.

[9] P. Kersten, J.-S. Lee, and T. Ainsworth, "Unsupervised classification of polarimetric synthetic aperture radar images using fuzzy clustering and em clustering," Geoscience and Remote Sensing, IEEE Transactions on, vol. 43, no. 3, pp. 519-527, March 2005.

[10] F. Barbaresco, "Interactions between symmetric cone and information geometries: Bruhat-tits and siegel spaces models for high resolution autoregressive doppler imagery," in Emerging Trends in Visual Computing, ser. Lecture Notes in Computer Science, F. Nielsen, Ed. Springer Berlin / Heidelberg, 2009, vol. 5416, pp. 124-163. [Online]. Available: http:/www.springerlink.com/content/654u7104744567w8/

[11] A. Alonso-Gonzalez, C. Lopez-Martinez, and P. Salembier, "Filtering and segmentation of polarimetric SAR data based on binary partition trees," IEEE Trans. Geoscience and Remote Sensing, vol. 50, no. 2, pp. $593-605$, feb. 2012.

[12] A. Alonso-González, C. López-Martínez, and P. Salembier, "Binary partition tree as a polarimetric SAR data representation in the space-time domain," in Geoscience and Remote Sensing Symposium (IGARSS), 2011 IEEE International, july 2011, pp. $3819-3822$.

[13] S. Valero, P. Salembier, and J. Chanussot, "Hyperspectral image segmentation using binary partition trees," in Image Processing (ICIP), 2011 18th IEEE International Conference on, 2011, pp. 1273-1276.

[14] - "Hyperspectral image representation and processing with binary partition trees," IEEE TIP, vol. 22, no. 4, pp. 1430-1443, 2013.

[15] — " "Object recognition in urban hyperspectral images using binary partition tree representation," in Geoscience and Remote Sensing Symposium (IGARSS), 2013 IEEE International, 2013, pp. 4098-4101.

[16] _ "Comparison of merging orders and pruning strategies for binary partition tree in hyperspectral data," in Image Processing (ICIP), 2010 17th IEEE International Conference on, 2010, pp. 2565-2568. 